\title{
Home Medication Review by Hospital Tuanku Fauziah: Carers' Perspectives
}

\author{
Wei Chern Ang ${ }^{1,2}$, Jurisma Che Lah $^{2}$, Nursyafiqah Zulkepli², Nursyamimi \\ Sukri $^{2}$, Amalina Rosedi ${ }^{2}$ \\ ${ }^{1}$ Clinical Research Centre, Hospital Tuanku Fauziah, Ministry of Health Malaysia, Perlis; \\ ${ }^{2}$ Department of Pharmacy, Hospital Tuanku Fauziah, Ministry of Health Malaysia, Perlis \\ DOI: http://dx.doi.org/10.31344/ijhhs.v5i0.315
}

Introduction: Home Medications Review (HMR) is a continuation of patient care from health facilities to their home to assess patients' pharmacotherapy by a multidisciplinary team. Bedridden patients were the main group who received this service. To improve the provision of HMR, we need to understand carers' viewpoints of the current service.

Objectives: To explore the carers' perspectives of HMR conducted by the medical outreach team (MOT) of Hospital Tuanku Fauziah.

Methods: This is a qualitative study conducted among carers who were involved in the HMR programme for more than six months. Subjects were recruited by purposive sampling from August 2019 to December 2019. In-depth interviews were audio-recorded at patients' homes until data saturation and transcribed verbatim. The transcripts then underwent thematic data analysis.

Results: Nine carers were interviewed. All participants had a limited understanding of HMR since they were not properly counselled prior to admission to the programme. The convenience of not having to go to the hospital was perceived as the major benefit of the programme. Healthcare providers were welcomed during each visit. Recognising allied health professionals in the MOT possesses a problem for some carers. There was a concern about having to collect newly add-on medications from the hospital. Some participants suggested increasing the frequency of visits and hope for more financial aids.

Conclusion: This study proved that carers' understanding of HMR was generally poor. All carers were satisfied with the current HMR programme provided by the MOT. However, several aspects of our HMR need to be improved. Despite the COVID-19 situation that puts HMR onhold and telemedicine have been adapted, HMR is here to stay in the post-COVID-19 era. This is supported by studies conducted in the pre-COVID-19 era that HMR is more beneficial than telemedicine due to the personal touch of face-to-face encounters.

Keywords: home care services, allied health personnel, caregivers, qualitative research, COVID-19 